\title{
Filament Eruptions Outside of Active Regions as Sources of Large Solar Energetic Particle Events
}

\author{
Stephen Kahler* \\ Air Force Research Laboratory \\ E-mail: stephen.kahlerekirtland.af.mil
}

\section{Nat Gopalswamy}

NASA Goddard Space Flight Center

\section{Pertti Makela, Sachiko Akiyama, Seiji Yashiro, Hong Xie, Neeharika Thakur}

NASA Goddard Space Flight Center and The Catholic University of America

\begin{abstract}
Gradual solar energetic $(E>10 \mathrm{MeV})$ particle (SEP) events are produced in shocks driven by fast CMEs, which are nearly always spatially associated with ARs. Several cases of SEP events associated with CMEs originating in large filament eruptions (FEs) from outside ARs have previously been known, but four more such cases from solar cycles 23 and 24 have been described by [1]. The CMEs were fast $\left(\sim 1000 \mathrm{~km} \mathrm{~s}^{-1}\right)$, appeared as coronagraph halo CMEs, and were associated with interplanetary type II bursts over a wide wavelength range. On the basis of their observed weak post-eruption arcade X-ray flare enhancements, several more candidate large SEP events resulting from eruptions of filaments adjacent to but outside ARs were identified. Thus, large SEP events can arise not only from unobserved ARs behind the disk, but also from non-AR filament eruptions. SEP event forecasting, currently based on observations of front-side solar ARs and X-ray flares, therefore can not predict either kind of SEP event. For the two SEP events with STEREO observations we confirm that despite their good magnetic connections to Earth, the SEP longitudinal distributions are broad. Neither Ulysses SEP observations nor CMEs associated with shocks and type II bursts give any indication that high-latitude polar-crown filament eruptions may have produced SEP events.
\end{abstract}

The 34th International Cosmic Ray Conference

30 July- 6 August, 2015

The Hague, The Netherlands

\footnotetext{
* Speaker.
} 


\section{Introduction}

Large gradual SEP $(E>10 \mathrm{MeV})$ events observed in the interplanetary medium are produced in shocks driven by fast $\left(>900 \mathrm{~km} \mathrm{~s}^{-1}\right)$ coronal mass ejections (CMEs) [2, 3, 4]. A major challenge is to forecast the occurrence or peak intensity Ip of a SEP event [5]. This is currently done for about a day in the future on the basis of the magnetic characteristics of ARs, which are assumed to provide the energy sources for the CMEs [6]. Fast CMEs are usually accompanied by AR flares, which can be exploited for immediate SEP forecasting, but only if those ARs lie on the visible solar disk. The accompanying AR flares are usually large, in the M or X range of the GOES soft X-ray scale. The time-intensity profiles of those X-ray flares are more easily and immediately analyzed for SEP forecasting than are the temporal and spatial variations of the white-light CME images, and the X-ray flare source locations can now be rapidly ascertained. Although less directly related to SEP production than are CMEs [7], flare observations continue to be the primary means of the immediate SEP forecasts [8].

In three cases large SEP events have been related to eruptions of filaments lying outside ARs and accompanied by only faint X-ray enhancements from the post-eruptive arcades that follow the filament eruptions (FEs) [9]. These few events are important not only for their elusive nature in the $\mathrm{AR}$ and flare-based SEP forecasting schemes, but also for their diagnostic insights into the physics of shock and SEP generation. In a recent work we [1] report four more such FE SEP events from solar cycles 23 and 24 and describe their characteristics in detail using complete data coverage from the Sun to the interplanetary medium. That work relates the FE properties to the associated CMEs and shocks. Here we will summarize and somewhat extend the findings [1] and then discuss the space weather implications of these events.

\section{Data Analysis}

\subsection{SEP Event Selection and Characteristics}

[1] selected only large SEP events with a proton intensity in the $>10 \mathrm{MeV}$ GOES energy channel $\geq 10 \mathrm{pfu}$ (pfu = particle flux unit; $1 \mathrm{pfu}=1$ particle $\mathrm{cm}^{-2} \mathrm{~s}^{-1} \mathrm{sr}^{-1}$ ). In addition, SEPs had to be detected in the $>50 \mathrm{MeV}$ channel for a fair comparison with previous events reported by [9]. Table 1 provides an overview of the four events, all of which have been listed in various papers involving statistical properties of SEP events or type II radio bursts [10, 4, 11]. The first two columns give the onset date and the peak intensity (Ip) of the SEP events in the GOES $>10 \mathrm{MeV}$ channel. Column 3 gives the best-fit exponent $\gamma$ to a power-law fit to the SEP energy spectrum at maximum intensity. Column 4 gives the heliographic coordinates of the filament centroid, and column 5 the soft X-ray flare size. Columns 6 and 7 give the speeds of the CME filament/prominences, Vep, and leading edges, $\mathrm{Vcme}$, observed in the LASCO field of view. The wavelength range of the type II burst is given in column 8; this range is a good indicator of where the shock forms and how long it survives [10]. Only one event had a type II burst starting from metric (m) wavelengths (starting frequency was $24 \mathrm{MHz}$ ). In all other cases, the type II burst started in the decameter-hectometric (DH) domain (below $13 \mathrm{MHz}$ ) as observed by the Radio and Plasma Wave Experiment (WAVES; [12]) on board the Wind spacecraft. In all cases, the radio emission continued down to kilometric (k) wavelengths, indicating that the shocks survived at least to $1 \mathrm{AU}$. 
Table 1: Large SEP Events With Prominence Eruption CMEs

\begin{tabular}{lrcccccc}
\hline \hline $\begin{array}{l}\text { SEP Onset } \\
\text { yyyy/mm/dd }\end{array}$ & $\begin{array}{r}\text { Ip } \\
\text { pfu }\end{array}$ & $\gamma$ & $\begin{array}{c}\text { Source } \\
\text { Location }\end{array}$ & $\begin{array}{c}\text { X-ray } \\
\text { Imp. }\end{array}$ & $\begin{array}{c}\text { Vep } \\
\mathrm{km} / \mathrm{s}\end{array}$ & $\begin{array}{c}\text { Vcme } \\
\mathrm{km} / \mathrm{s}\end{array}$ & $\begin{array}{c}\text { type II } \\
\mathrm{m} / \mathrm{Dk}\end{array}$ \\
\hline $2000 / 09 / 12$ & 320 & 4.15 & S17W09 & M1.0 & 677 & 1550 & $\mathrm{y} / \mathrm{Y}$ \\
$2002 / 05 / 22$ & 820 & 4.55 & S30W34 & C5.0 & 621 & 1494 & $\mathrm{n} / \mathrm{Y}$ \\
$2011 / 11 / 26$ & 80 & 4.34 & N27W49 & C1.2 & 878 & 933 & $\mathrm{n} / \mathrm{Y}$ \\
$2013 / 09 / 29$ & 180 & 4.69 & N23W25 & C1.1 & 603 & 1025 & $\mathrm{n} / \mathrm{Y}$ \\
Additional & \multicolumn{7}{c}{ Events } \\
\hline $2000 / 04 / 04$ & 55 & 4.76 & N25W55 & C9.7 & & & \\
$2004 / 04 / 11$ & 35 & 4.01 & S14W49 & C9.6 & & 1188 & $\mathrm{y} / \mathrm{Y}$ \\
\hline
\end{tabular}
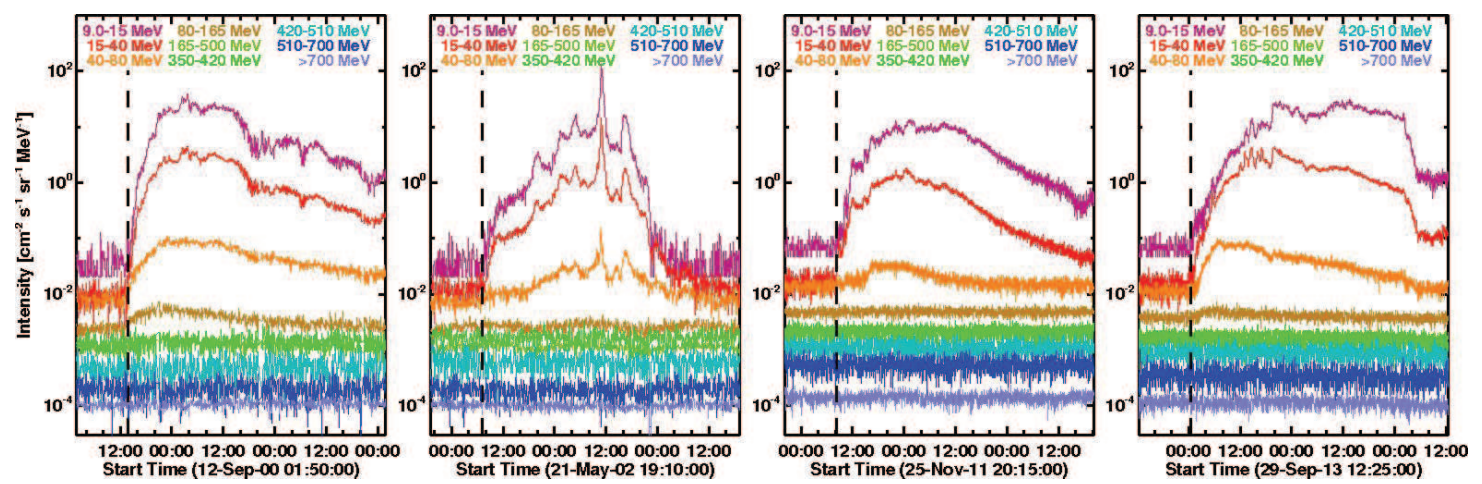

Figure 1: Differential proton intensity profiles of the first four SEP events of Table 1. Figure 2 of [1].

The four SEP events had proton intensity increases extending to $50 \mathrm{MeV}$, and their profiles in the GOES observations are shown in Figure 1. [1] used proton intensities observed by the Energetic and Relativistic Nuclei and Electron (ERNE; [13]) instrument on board SOHO to derive the powerlaw spectral index $\gamma$, which ranges from 4.15 to 4.69 (Table 1), comparable to the 4.3 value derived for the 1981 December 5 SEP event [14]. These values also lie in the high end of the approximate range of $2<\gamma<4.5$ for well-connected $>10$ pfu SEP events of the later GOES two-point $(E>$ $10 \mathrm{MeV}$ and $>60 \mathrm{MeV}$ ) survey by [15].

\subsection{Solar Source Observations}

In the pre-eruption phase, the source regions were large-scale bipolar regions with $\mathrm{H} \alpha$ filaments marking the polarity inversion lines of the magnetic structures, as shown in the top panels of Figure 2. The filament eruption was accompanied by a two-ribbon flare in each case, with the ribbons located on either side of the original position of the filament. The photospheric magnetograms of Figure 2 show that the flare ribbons were located on opposite polarity regions, while the filaments were located on the polarity inversion line. The ribbons in the 2011 November 26 event were confined to the stronger magnetic field region, where the pre-eruption filament was very thin. There was another section of the filament that erupted from the southern end, but no ribbons were found around this section. The post-eruption arcades of Figure 2 were also very extended, mostly in the north-south direction. The soft X-ray flares associated with the four SEP events were 

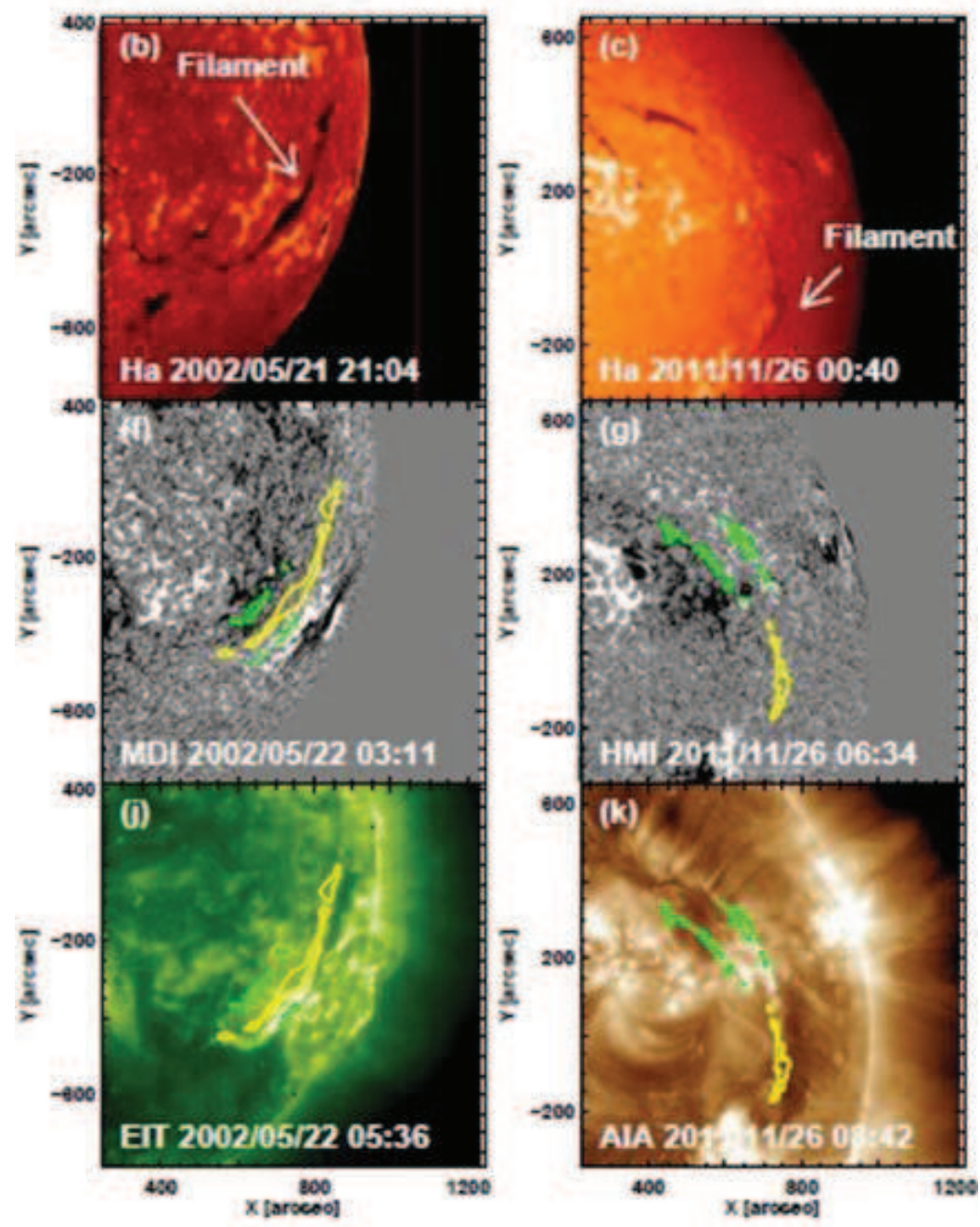

Figure 2: Images of the second and third events of Table 1. The filaments in H $\alpha$ images (top); flare ribbons (green) and filament locations (yellow) on magnetograms (middle); EUV post-eruption arcades (bottom). From [1].

generally weak, with the flare size ranging from C1.2 to M1.0. The M1.0 flare associated with the 2000 September 12 event was the only one with weak impulsive microwave emission.

The later phases of the erupting filaments were observed as prominences trailing the CME leading edges in the LASCO field of view, shown for the four events in Figure 3 [1]. The four height-time plots showed the well known [16] effect of separations between the CME leading edges and prominences because of their different speeds (Table 1). The CMEs all met the criteria for fast $\left(\geq 900 \mathrm{~km} \mathrm{~s}^{-1}\right)$ and wide $\left(\geq 60^{\circ}\right)$ to drive shocks and accelerate SEPs, but the CME leading edge accelerations were found to be relatively low in comparison with those of large SEP events [17].

In all events, the type II bursts were weak and of narrow band in the DH domain, but intensified around $1 \mathrm{MHz}$. Shock speeds were deduced from the type II burst drift rates, yielding values comparable to those of the associated CME leading edge speeds, suggesting that the SEP events 


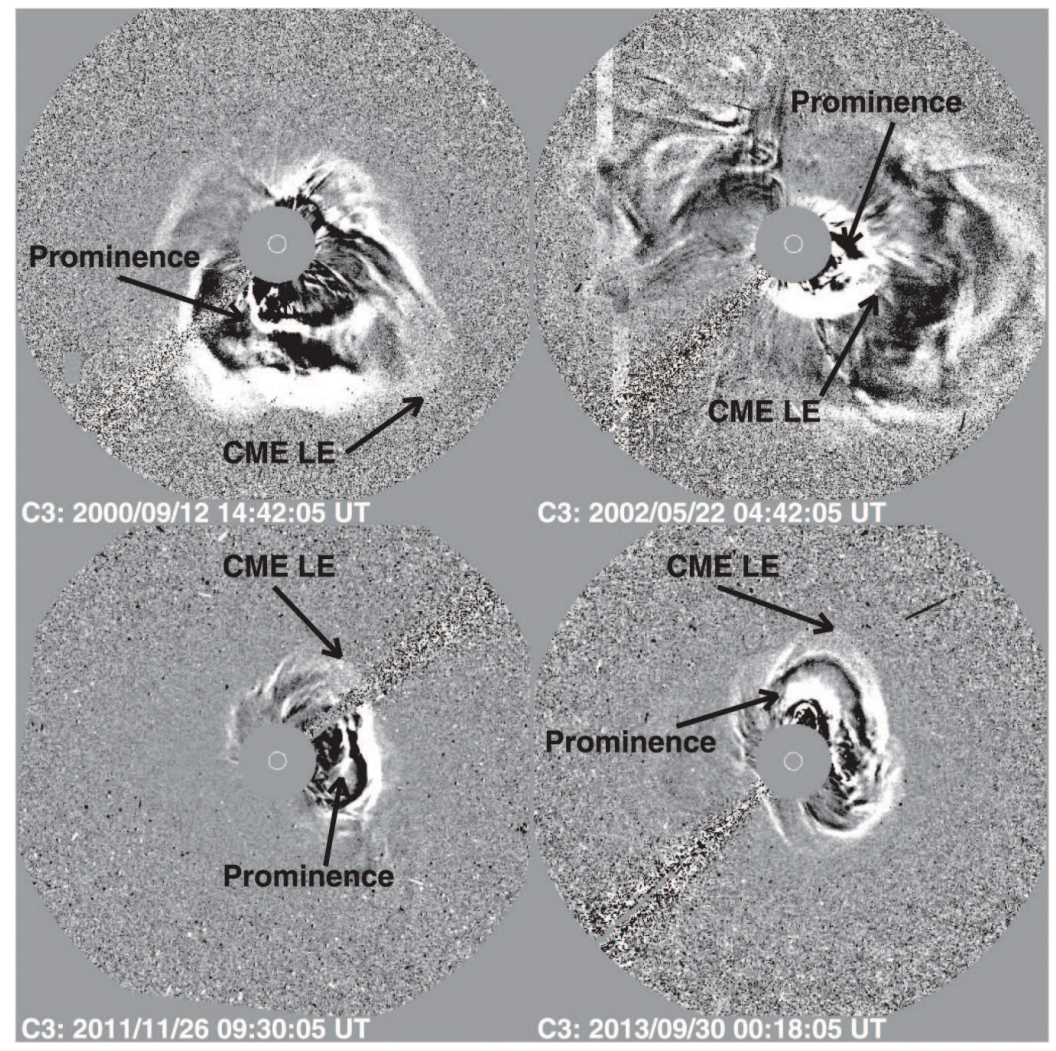

Figure 3: SOHO/LASCO images showing the CMEs and the associated eruptive prominences in four SEP events. All four CMEs became halos in the field of view of the outer coronagraph (C3) of SOHO/LASCO. Figure 5 of [1].

were consistent with shock acceleration. The general lack of a metric type II component (Table 1) indicates that the associated shocks formed relatively late. Using previous determinations of shock formation heights [18], [1] established formation heights of $\geq 1.92$ Rs. All the shocks were relatively strong and survived to the Earth, where they were observed in situ as interplanetary shocks.

\subsection{Search for Additional FE SEP Events}

[1] used the typical characteristics of FE SEP events to search for additional such candidates among SEP events for which AR sources had been assumed or assigned. They selected large ( $>10$ pfu) SEP events over cycles 23 and 24 with weak C-class flares and found 5 candidate events. The two best cases of FE events are included at the bottom of Table 1. Examination of H $\alpha$, EUV, and magnetogram data showed that a north-south $\mathrm{FE}$ and post-eruption arcade occurred $\sim 10^{\circ}$ to the east of AR 8933 on 2000 April 4. A circular filament mostly outside AR 0588 was the source region for the 2004 April 11 SEP event and had been studied in detail earlier by [19]. [1] concluded that the 2000 April 4 event was definitely and the 2004 April 11 event likely an FE SEP event. Table 1 shows that they are similar in all characteristics to the first four events, so there now may be 6 additional FE events to add to the earlier list. The remaining questionable cases suggest a 
continuum of filament-AR configurations from the more usual filaments embedded in ARs to the clearly non-AR filaments of the first four cases of Table 1.

\subsection{SEP Longitudinal Extensions of Two EF Events.}

The source longitudes of the 6 EF SEP events of Table 1 are all magnetically well connected to Earth. Including the remaining three candidate SEP events with C-class flares, the 9 EF sources range from $\mathrm{W} 09^{\circ}$ to $\mathrm{W} 66^{\circ}$, reminiscent of the limited longitude range of impulsive SEP events [2, $20,21]$ and their narrow longitudinal SEP distributions. For events since 2006, STEREO observations can provide spatial distributions of gradual SEP events. We have compared the two latest SEP events of Table 1 with the catalog of STEREO and Earth-based $\sim 25 \mathrm{MeV}$ peak proton intensities Ip compiled by [22]. Both events were observed at all three locations, and the maximum Ip was at Earth. The four gradients (two directions and two events) of log Ip versus longitude in degrees ranged from 0.017 to $0.001 \mathrm{deg}^{-1}$. These gradients are comparable to those on the log plot of Ip versus longitude for 15-40 MeV protons of 35 SEP events observed at STEREO and Earth by [23]. Thus, despite the favorable magnetic source connections of the Table-1 EF SEP events, the longitudinal distributions are most likely comparable to those of more typical SEP events.

\subsection{High Latitude SEP Events and Polar Crown Filaments}

An extreme example of EFs is that of the high-latitude polar crown filaments, which lie well above the AR zone latitudes. Could polar crown EFs give rise to SEP events? High latitude SEP events were observed on the polar passes of the Ulysses spacecraft, so we examined works dealing with Ulysses SEP events [24, 25, 26] to determine the source regions of those events. In all cases those SEP events could be attributed to CMEs/flares in ARs. The other approach is to ask whether polar crown EFs and CMEs can be associated with type II bursts, indicating that they can drive shocks. Selecting only fast $\left(>900 \mathrm{~km} \mathrm{~s}^{-1}\right.$ ) and wide $\left(\geq 60^{\circ}\right)$ (FW) CMEs from 1996 to 2005, [27] found that solar sources of CMEs both with and without any type II bursts were confined generally to a $\pm 40^{\circ}$ latitude range. A later survey [28] of CMEs associated with interplanetary shocks detected at Earth revealed a CME source latitude range of $\pm 30^{\circ}$. In both studies, there was a pattern of the highest latitudes occurring during the solar cycle rise phase, consistent with the AR latitudes. We conclude that neither the Ulysses SEP observations nor the FW and shock-associated CME studies give any indication that polar crown EFs produce SEP events.

\section{Discussion}

Most large SEP events are associated with fast and wide CMEs from solar ARs with M or X class flares. However, the SEP events associated with EFs outside of ARs (GMA2015, [1]) are a small but well defined class of events that can be difficult to forecast when the AR X-ray flare is the basic forecasting diagnostic. These SEP events differ primarily in that the CME accelerations are lower, resulting in later shock formation at greater coronal heights. After formation, however, the shocks can be strong and propagate to the Earth. Perhaps the late onset of SEP acceleration is the reason for their characteristically steep energy spectra. The EF SEP events found so far are nearly all magnetically connected to Earth, but they probably have longitudinal intensity peaks similar to AR-associated SEP events. These EF SEP events appear to be confined to AR latitude sources. 


\section{Acknowledgements}

S. Kahler was funded by AFOSR Task 15RVCOR167. CME data were taken from the CDAW LASCO catalog. This CME catalog is generated and maintained at the CDAW Data Center by NASA and The Catholic University of America in cooperation with the Naval Research Laboratory. SOHO is a project of international cooperation between ESA and NASA.

\section{References}

[1] N. Gopalswamy, P. Mäkelä, S. Akiyama, S. Yashiro, H. Xie, N. Thakur, and S.W. Kahler, Large Solar Energetic Particle Events Associated with Filament Eruptions Outside of Active Regions, Astrophys. J., 806, (2015), 8 .

[2] D.V. Reames, Particle acceleration at the Sun and in the heliosphere, Space Sci. Rev., 90, (1999), 413.

[3] D.V. Reames, The Two Sources of Solar Energetic Particles, Space Sci. Rev., 175, (2013), 53.

[4] N. Gopalswamy, H. Xie, S. Akiyama, P.A. Mäkelä, and S. Yashiro, Major solar eruptions and high-energy particle events during solar cycle 24, Earth, Planets and Space, 66, (2014), 104.

[5] C.C. Balch, Updated verification of the Space Weather Prediction Center's solar energetic particle prediction model, Space Weather, 6, (2008), S01001.

[6] D.A. Falconer, R.L. Moore, A.F. Barghouty, and I. Khazanov, Prior Flaring as a Complement to Free Magnetic Energy for Forecasting Solar Eruptions, Astrophys. J., 757, (2012), 32.

[7] J. Park, Y.-J. Moon, and N. Gopalswamy, Dependence of solar proton events on their associated activities: Coronal mass ejection parameters, J. Geophys. Res., 117, (2012), A08108.

[8] S.W. Kahler, A. Ling, and S.M. White, Forecasting SEP Events with Same Active-Region Prior Flares, Space Weather, in press, (2015).

[9] S.W. Kahler, Origin and Properties of Solar Energetic Particles in Space, in Space Weather, AGU Geophysical Monograph 125, (2000), 109.

[10] N. Gopalswamy, E. Aguilar-Rodriguez, S. Yashiro, N. Nunes, M.-L. Kaiser, and R.A. Howard, Type II radio bursts and energetic solar eruptions, J. Geophys. Res., 110, (2005), A12S07.

[11] N. Gopalswamy, Energetic particle and other space weather events of solar cycle 24, in: Space Weather: The Space Radiation Environment: 11th Annual International Astrophysics Conference. AIP Conf. Proc. 1500, (2012), 14.

[12] J.-L. Bougeret, et al., Waves: The Radio and Plasma Wave Investigation on the Wind Spacecraft, Space Sci. Rev., 71, (1995), 231.

[13] J. Torsti, E. Valtonen, M. Lumme, et al., Energetic Particle Experiment ERNE, Solar Phys., 162, (1995), 505.

[14] S.W. Kahler, E.W. Cliver, H.V. Cane, R.E. McGuire, R.G. Stone, and N.R. Sheeley, Jr., Solar filament eruptions and energetic particle events, Astrophys. J., 302, (1986), 504.

[15] S.W. Kahler, The correlation between solar energetic particle peak intensities and speeds of coronal mass ejections: Effects of ambient particle intensities and energy spectra, J. Geophysical Res., 106, (2001), 20947.

[16] N. Gopalswamy, M. Shimojo, W. Lu, S. Yashiro, K. Shibasaki, and R.A. Howard, Prominence Eruptions and Coronal Mass Ejection: A Statistical Study Using Microwave Observations, Astrophys. J., 586, (2003), 562. 
[17] P. Mäkelä, N. Gopalswamy, S. Akiyama, H. Xie, and S. Yashiro, Estimating the Height of CMEs Associated with a Major SEP Event at the Onset of the Metric Type II Radio Burst during Solar Cycles 23 and 24, Astrophys. J., 806, (2015), 13.

[18] N. Gopalswamy, H. Xie, P. Mäkelä, et al., Height of shock formation in the solar corona inferred from observations of type II radio bursts and coronal mass ejections, Adv. Space Res., (2013), 51, 1981.

[19] N. Gopalswamy and P. Mäkelä, Long-duration Low-frequency Type III Bursts and Solar Energetic Particle Events, Astrophys. J. Let., 721, (2010), L62.

[20] Y.-M. Wang, M. Pick, and G.M. Mason, Coronal Holes, Jets, and the Origin of ${ }^{3}$ He-rich Particle Events, Astrophys. J., 639, (2006), 495.

[21] N.V. Nitta, D.V. Reames, M.L. DeRosa, Y. Liu, S. Yashiro, and N. Gopalswamy, Solar Sources of Impulsive Solar Energetic Particle Events and Their Magnetic Field Connection to the Earth, Astrophys. J., 650, (2006), 438.

[22] I.G. Richardson, T.T. von Rosenvinge, H.V. Cane, E.R. Christian, C.M.S. Cohen, A.W. Labrador, R.A. Leske, R.A. Mewaldt, M.E. Wiedenbeck, and E.C. Stone, > $25 \mathrm{MeV}$ Proton Events Observed by the High Energy Telescopes on the STEREO A and B Spacecraft and/or at Earth During the First Seven Years of the STEREO Mission, Solar Phys., 289, (2014), 3059.

[23] D. Lario, A. Aran, R. Gómez-Herrero, N. Dresing, B. Heber, G.C. Ho, R.B. Decker, and E.C. Roelof, Longitudinal and Radial Dependence of Solar Energetic Particle Peak Intensities: STEREO, ACE, SOHO, GOES, and MESSENGER Observations, Astrophys. J., 767, (2013), 41.

[24] R.B. McKibben, J.J. Connell, C. Lopate, M. Zhang, J.D. Anglin, A. Balogh, S. Dalla, T.R. Sanderson, R.G. Marsden, M.Y. Hofer, H. Kunow, A. Posner, and B. Heber, Ulysses COSPIN observations of cosmic rays and solar energetic particles from the South Pole to the North Pole of the Sun during solar maximum, Annal. Geophys., 21, (2003), 1217.

[25] S. Dalla, A. Balogh, S. Krucker, A. Posner, R. Müller-Mellin, J.D. Anglin, M.Y. Hofer, R.G. Marsden, T.R. Sanderson, C. Tranquille, B. Heber, M. Zhang, and R.B. McKibben, Properties of high heliolatitude solar energetic particle events and constraints on models of acceleration and propagation, Geophys. Res. Let., 30, (2003), 8035.

[26] T.R. Sanderson, Propagation of Energetic Particles to High Latitudes, in The Sun and Heliosphere as an Integrated System, Kluwer Publishers, Dordrecht, (2004), 113

[27] N. Gopalswamy, S. Yashiro, H. Xie, S. Akiyama, E. Aguilar-Rodriguez, M.L. Kaiser, R.A. Howard, and J.-L. Bougeret,Radio-Quiet Fast and Wide Coronal Mass Ejections, Astrophys. J., 674, (2008), 560 .

[28] N. Gopalswamy, H. Xie, P. Mäkelä, S. Akiyama, S. Yashiro, M.L. Kaiser, R.A. Howard, and J.-L. Bougeret, Interplanetary Shocks Lacking Type II Radio Bursts, Astrophys. J., 710 (2010), 1111. 